\title{
Orthogonality in normed linear spaces: a classification of the different concepts and some open problems
}

\author{
CARLOS BENITEZ
}

\begin{abstract}
Orthogonality in inner product spaces is a binary relation that can be expressed in many ways without explicit mention to the inner product of the space.

Great part of such definitions have also sense in normed linear spaces. This simple observation is at the base of many concepts of orthogonality in these more general structures.

Various authors introduced such concepts over the last fifty years, although the origins of some of the most interesting results that can be obtained for these generalized concepts are, as usual in Mathematics, in previous or parallel works about convex sets, ellipsis or ellipsoids, duality, etc. (see, e.g., Gruber's paper [13] about the prior contributions of Caratheodory, Blaschke or Radon).
\end{abstract}

\section{DIFFERENT CONCEPTS OF ORTHOGONALITY}

According to its greater frecuency in the literature this exposition is limited to the case in wich $\mathrm{E}$ is a real normed linear space.

When the norm of $E$ is induced by an inner product, the orthogonality of two points $x$ and $y$ of $E$ is equivalent to each one of next (main or secondary) propositions.

In the more general context of normed linear spaces any one of such propositions is a definition of orthogonality between $x$ and $y$.

ROBERTS (1934): $\|x-\lambda y\|=\|x+\lambda y\|$, for every $\lambda \in \mathbb{R}$.

BIRKHOFF (1935): $\|x\| \leq\|x+\lambda y\|$, for every $\lambda \in \mathbb{R}$.

CARLSSON (1961): $\sum_{k=1}^{m} a_{k}\left\|b_{k} x+c_{k} y\right\|^{2}=0$, where $m \geq 2$ and $a_{k}, b_{k}, c_{k}$ are real numbers such that

1980 Mathematics Subject Classification (1985 revision): 46B20

Editorial de la Universidad Complutense. Madrid, 1989. 


$$
\sum_{k=1}^{m} a_{k} b_{k} c_{k} \neq 0, \sum_{k=1}^{m} a_{k} b_{k}^{2}=\sum_{k=1}^{m} a_{k} c_{k}^{2}=0
$$

Obviously C-orthogonality is not a single concept of orthogonality but a family of them. Before and after Carlsson's paper [8] the following members of such family have been considered separately:

Isosceles (1945): $\|x-y\|=\|x+y\|$

Pythagorean (1945): $\|x-y\|^{2}=\|x\|^{2}+\|y\|^{2}$

both introduced by James [14].

a-Isosceles (1988): $\|x-a y\|=\|x+a y\|$

a-Pythagorean (1988): $\|x-a y\|^{2}=\|x\|^{2}+a^{2}\|y\|^{2}$

both for some fixed $a \neq 0$. They appear in [3] starting from a hint of [6].

ab (1978): $\|a x+b y\|^{2}+\|x+y\|^{2}=\|a x+y\|^{2}+\|x+b y\|^{2}$

where $a, b \in(0,1)$, considered by Kapoor and Prasad [18].

a (1983): $\left(1+a^{2}\right)\|x+y\|^{2}=\|a x+y\|^{2}+\|x+a y\|^{2}$

where $a \neq 1$, considered by Diminnie, Freese and Andalafte [12].

UNITARY-CARLSSON: either $\|x\|\|y\|=0$, or $\|x\|^{-1} x$ is Carlsson-orthogonal to $\|y\|^{-1} y$.

Apparently there is no general study of this family of concepts. However, as for Carlsson's one, some particular members of this family have been considered:

U-Isosceles (1957): either $\|x\|\|y\|=0$, or $\|x\|-1$ is Isosceles-orthogonal to $\|y\|^{-1} y$; introduced by Singer [22].

U-Pythagorean (1986): either $\|x\|\|y\|=0$, or $\|x\|^{-1} x$ is Pythagorean-orthogonal to $\|y\|^{-t} y$, considered by Diminnie, Andalafte and Freese [11].

DIMINNIE (1983): $\sup \left(f(x) g(y)-f(y) g(x): f, g \in S^{\prime}\right\}=\|x\|\|y\|$, where $S^{\prime}$ denotes the unit sphere of the topological dual of $E$.

AREA (1984): either $\|x\|\|y\|=0$ or they are linearly independent and such that $x, y,-x,-y$ divide the unit ball of their own plane (identified to $\mathbb{R}^{2}$ ) in four equal areas. Introduced by Alonso [1]. 
As we have pointed out above all these propositions mean the same (orthogonality between $x$ and $y$ ) when $\mathrm{E}$ is an inner product space, but, in general, they do not mean the same in normed linear spaces. Moreover, equivalence between almost any two of them is a characteristic property of inner product spaces. $[1,2,9,17,20]$.

On the other hand, orthogonality in inner product spaces is symmetric ( $x \perp y$ implies $y \perp x)$, homogeneous $(x \perp y$ and $\lambda, \mu \in \mathbb{R}$ imply $\lambda x \perp \mu y)$, additive ( $x \perp y$ and $x \perp z$ imply $x \perp y+z$ ), existing (for every $x, y \in \mathrm{E}$ there exists $\alpha \in \mathbb{R}$ such that $x \perp a x+y$ ), unique (the above $a$ is unique when $x \neq 0$ ), etc.

The study of such properties for generalized orthogonalities in normed linear spaces is one of the main topics of many papers. A description of current knowledge with an extensive bibliography can bee seen in [2].

\section{SOME OPEN PROBLEMS}

Within the two families of the above-mentioned questions (relations between two different orthogonalities and properties of each orthogonality) some substantial problems remain open. Among them some of the most general and, perhaps, most interesting are the following ones:

Problem 1. It is known, e.g., that if Isosceles implies Pythagorean, or conversely, then $E$ is an inner product space [9]. However nothing similar seems to be know for the general case of any two C-orthogonalities.

We conjecture that if a $C$-orthogonality implies another "essentially different" C-orthogonality then $E$ is an inner product space.

Problem 2. It is known, e.g., that $\mathrm{U}$-Isosceles is equivalent to U-Pythagorean when $E$ is the space $\mathbb{R}^{2}$ endowed with a norm whose unit sphere is a regular octogon [1]. It is also known for other pairs of U-orthogonalities (even in two dimensional spaces) that if one implies the other then $E$ is an inner product space [3].

Our conjecture is that if dimE $=3$ and if an U-orthogonality implies another "esentially different" $U$-orthogonality then $E$ is an inner product space.

Problem 3. There are C-orthogonalities that are trivially symmectric (Isosceles, Pythagorean, ...) and other that are non symmetric (a-Isosceles, a-Pythagorean, with $a \neq 1, \ldots)$. Moreover, it is known that if a-Isosceles or a-Pythagorean $(a \neq 1)$ are symmectric then $\mathrm{E}$ is an inner product space $[18,19]$. 
We conjecture that either a C-orthogonality is "trivially symmetric" or such a property is characteristic of inner oproduct spaces.

Problem 4. It is known that additivity of Carlsson (on the left or on the right in non-symmetric cases) is equivalent to homogeneity and a characteristic property of inner product spaces [8].

It is known also that additivity of Diminnie [10], U-Pythagorean [7] and Birkhoff (on the left) [15] are characteristic properties of inner product spaces of dimension $\geq 3$. But additivity on the right of Birkhoff is characteristic of smooth spaces [16] and additivity of Roberts may be trivial since this orthogonality may be strongly non-existing (i.e. such that $x \perp y$ if and only if $\|x\|\|y\|=0)[14]$.

On the other hand, in two-dimensional spaces and for homogeneous orthogonalities (all of them excepting Carlsson), additivity is equivalent to uniqueness and hence it is either a general property of some orthogonalities as Area, U-Isosceles, ..., (in 2-dimensional spaces) or it is closely related to some geometric properties of the space (rotundity, somoothness, ...) which are weaker than its norm induced by an inner product.

Our conjecture is that additivity of Area and U-Carlsson (on the left and on the right in non symmetric cases) are characteristic properties of inner product spaces of dimension $\geq 3$.

\section{References}

[1] J. Alonso. Ortogonalidad en espacios normados. Ph. D. Thesis, Universidad de Extremadura, 1984.

[2] J. ALONSO and C. BENITEZ. Orthogonality in normed linear spaces. A survey: Main properties. Extracta Math. 3 (1988) 1-15.

[3] J. ALONSO and C. BENITEZ. Some characteristic and non-characteristic properties of inner product spaces. J. Approx. Theory 55 (1988) 318-325.

[4] D. AMIR. Characterizations of Inner Product Spaces. Birkhäuser, 1986.

[5] G. BiRKHOFF. Orthogonality in linear metric spaces. Duke Math. J. 1 (1935) $169-172$.

[6] J. BORWEIN and L. KEENER. The Hausdorff metric and Chebyshev centers. J. Approx. Theory 28 (1980) 366-376.

[7] A. P. BOSZNAY. On a problem concerning orthogonality in normed linear spaces. Preprint.

[8] S.O. CaRLSSON. Orthogonality normed linear spaces. Ark. Mat. 4(1961) 297-318.

[9] M. M. DAY. Some characterizations of inner product spaces. Trans. Amer. Math. Soc. 62 (1947) 320-337. 
[10] C. R. DiminNIE. A new orthogonality relation in normed linear spaces. Math. Nachr. 114(1983) 197-203.

[11] C. R. DiminNiE, E. Z. ANDALAFTE and R. W. Freese. Angles in normed linear spaces and a characterization of real inner product spaces. Math. Nachr. 129 (1986) 197-204.

[12] C. R. Diminnie, R. W. Freese and E. Z. Andalafte. An extension of Pythagorean and Isosceles orthogonality and a characterization of inner product spaces. J. Approx. Theory 39 (1983) 295-298.

[13] P. M. Gruber. "4. Konvexität", in: Collected Works of Johann Radon, Volume 1. Edited by P.M. Gruber, E. Hlawka, W. Nōbauer and L. Schmetterer. Birkhäuser, 1987.

[14] R. C. JAMES. Orthogonality in normed linear spaces. Duke Math. J. 12(1945) 291-301.

[15] R. C. JAMES. Inner products in normed linear spaces. Bull. Amer. Math. Soc. 53 (1947) 559-566.

[16] R. C. JAMES. Orthogonality and linear functionals in normed linear spaces. Trans. Amer. Math. Soc. 61 (1947) 265-292.

[17] O. P. KAPOOR and S. B. MATHUR. Some geometric characterizations of inner product spaces. Bull. Aust. Math Soc. 24 (1981) 239-246.

[18] O. P. KAPOOR and J. PRASAD. Orthogonality and characterizations of inner product spaces. Bull. Aust. Math. Soc. 19 (1978) 403-416.

[19] E. R. LORCH. On certain implications which characterize Hilbert space. Ann. of Math. 49 (1948) 523-532.

[20] K. OHIRA. On some characterizations of abstract euclidean spaces by properties of orthogonality. Kumamoto J. Sci. A1 (1952) 23-26.

[21] B. D. ROBERTS. On the geometry of abstract vector spaces. Tôhoku Math. J. 39 (1934) 42-59.

[22] I. SINGER. Unghiuri abstracte si functii trigonometrice in spactii Banach. Bul. Sti. Acad. R.P.R., Sect. Sti. Mat. Fiz. 9 (1957) 29-42.

[23] I. SingER. Best Approximatión in Normed Linear Spaces by Elements of Linear Subspaces. Springer, 1970.

Departamento de Matemáticas

Universidad de Extremadura

06071 Badajoz (ESPAÑA) 